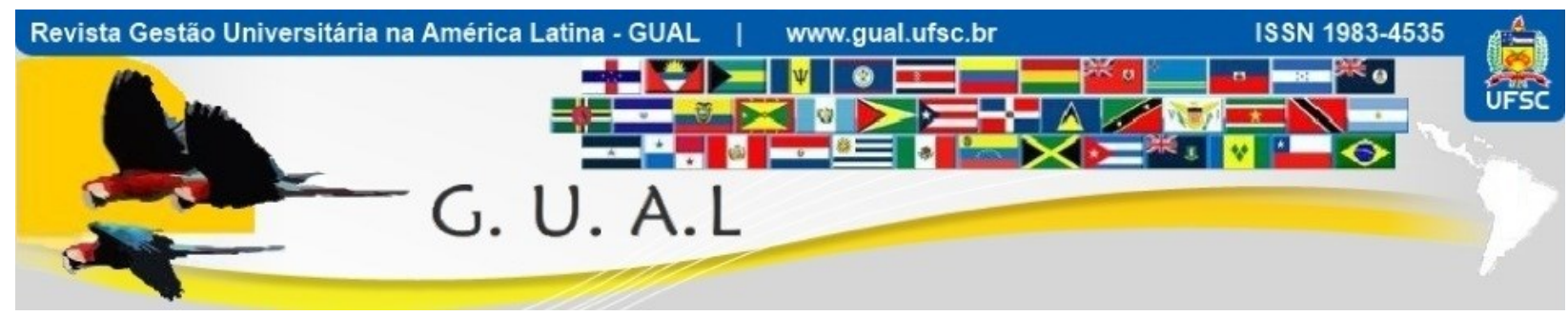

DOI: http://dx.doi.org/10.5007/1983-4535.2015v8n1p110

\title{
PROPUESTA TEÓRICO METODOLÓGICA PARA EL ANÁLISIS DE LAS COMPETENCIAS PROFESIONALES Y SU IMPACTO EN LA EMPLEABILIDAD
}

\author{
METHODOLOGICAL PROPOSAL FOR THEORETICAL ANALYSIS \\ PROFESSIONAL SKILLS AND ITS IMPACT ON EMPLOYABILITY
}

Rebeca Almanza Jiménez, Especialista Instituto tecnológico de Lázaro Cárdenas - ITLAC rebeca_aj@hotmail.com e itlac_aj@hotmail.com

José Guadalupe Vargas-Hernández, Ph.D. Universidad de Guadalajara - UDG jvargas2006@gmail.com,jigvh0811@yahoo.com, josevargas@cucea.udg.mx

Recebido em 02/fevereiro/2014

Aprovado em 26/novembro/2014

Sistema de Avaliação: Double Blind Review

Esta obra está sob uma Licença Creative Commons Atribuição-Uso. 


\begin{abstract}
RESUMEN
El objetivo del presente artículo es analizar el nivel de empleabilidad de los Ingenieros en Gestión Empresarial Egresados del Instituto tecnológico de Lázaro Cárdenas en función de sus competencias profesionales partiendo de la hipótesis inicial. Las competencias profesionales tienen una relación directa positiva en la empleabilidad lo que a través de la aplicación del método de encuesta en escala tipo Likert aplicada a ambos conglomerados, se realizó el análisis de los hallazgos los cuales evidencian el impacto de las competencias profesionales en la empleabilidad, la existencia de una correlación directa positiva de ambas y la oportunidad para desarrollar la creatividad e innovación mediante el emprendedurismo.
\end{abstract}

Palabras-clave: Competencias Profesionales. Egresados. Empleabilidad. Emprendedurismo.

\begin{abstract}
The objective of this paper is to analyze the level of employability of Engineers in Business Management Institute of Technology graduates Lazaro Cardenas in terms of their Professional competencies based on the initial hypothesis. The professional competencies have a direct positive relationship in which employability through the application of the survey method in Likert applied to both clusters, analysis of findings which show the impact of skills on employability, the existence of a positive correlation of both direct and opportunity was conducted to develop creativity and innovation through entrepreneurship.
\end{abstract}

Keywords: Professional Competencies. Graduates. Employability. Entrepreneurship. 


\section{INTRODUCCIÓN}

En la actualidad a las instituciones de educación superior se les demanda una mejor preparación de los jóvenes en el mundo laboral y el desarrollo de competencias profesionales que permitan la movilización de conocimientos a situaciones de trabajo. Molina (2007). Frente a las exigentes demandas de ingenieros calificados Rascón, (2010) y Meza, (2008) se hace necesario que los estudiantes cuenten con conocimientos acordes a su perfil de egreso, desarrollen habilidades y valores.

En el sector productivo se perfila la necesidad de desarrollo de proyectos de investigación que den solución a problemas de contexto, que demuestren los estudiantes la capacidad de trabajar en equipos multidisciplinarios; la capacidad para identificar, formular y resolver problemas de ingeniería; la comprensión de la responsabilidad profesional y ética; la capacidad de comunicarse eficazmente y una educación suficientemente amplia para comprender el impacto de las soluciones de ingeniería en un contexto global, económico, ambiental y social Paz, (2007).

El tema es de gran relevancia para la exploración de la articulación entre la formación de competencias profesionales que se ofrecen en el Instituto Tecnológico de Lázaro Cárdenas Michoacán (ITLAC) y las necesidades del mercado laboral. También es útil para ajustar e innovar el desempeño profesional de los egresados de la carrera de Ingeniería en Gestión Empresarial con las demandas del sector productivo y realizar una evaluación de la pertinencia y actualidad de los mismos.

Los resultados de este trabajo permiten revizar e identificar las competencias profesionales que optimicen y mejoren la inserción laboral de titulados y tituladas en puestos cualificados y mejoren la empleabilidad y competitividad en el mercado de trabajo actual. Asi pues, se analizan las necesidades actuales de las empresas empleadoras sobre las competencias profesionales que deben de tener los Ingenieros en Gestión Empresarial las cuales permiten potenciar en los proximos egresados los conocimientos, habilidades, destrezas y actitudes requeridas por el mercado laboral para que estos puedan desempeñar exitosamente diferentes funciones y actividades. Esta investigación se aborda en seis apartados interrelacionados que constituyen los distintos capítulos que la componen:

En base a lo anterior el objetivo del presente trabajo consiste en determinar el nivel de empleabilidad de los Ingenieros en Gestión Empresarial egresados del ITLAC en función de las competencias profesionales y se estructura iniciando con una breve introducción, seguida 
de los antecedentes y delimitación del problema, justificación, hipótesis, marco conceptual, marco contextual, propuesta, conclusiones y referencias.

\section{ANTECEDENTES EL PROBLEMA}

Las tendencias internacionales han demostrado que en las Instituciones de Educación Superior (IES) no existe pertinencia educativa, lo cual genera una brecha entre los conocimientos y destrezas de los egresados y las necesidades de un entorno laboral competitivo ( Delval, 2002; Gibbons, 1998; OCDE, 2000; Tünnermann, 2002; Tobón, 2005), razón por lo cual diversos sectores demandan a las IES una formación profesional acorde a los diferentes procesos productivos, mismos que exigen el desarrollo de nuevas necesidades y exigencias relativas a las competencias y conocimientos de los egresados que les permita insertarse activamente en el mundo laboral (ANUIES, 2003).

Como consecuencia del fenómeno de la sobre educación aunado a la imposibilidad del sistema económico de proporcionar puestos de trabajo acordes a las condiciones del capital humano, en México, actualmente el desempleo afecta al 40\% de los egresados universitarios, si la economía no aumenta su nivel de crecimiento en esta década 305,000 egresados de todo el país se enfrentarán a la desocupación en el 2020. En los últimos diez años, el número de profesionales en el país aumento $2.8 \%$ pero, de ese total, un $16 \%$ se mantuvo inactivo. La tasa de desempleo en el área profesional subió del 2.3\% al 5.1\%. ANUIES (2014).

Algunos de los factores que propician la existencia de esta problemática son: formación académica en las universidades orientada tradicionalmente a la búsqueda de empleo, poca investigación en ciencia aplicada, escasa vinculación universidad-empresa, pocos estímulos para la creación de empresas, ausencia de habilidades emprendedoras proporcionadas en la universidad, y baja preparación académica del personal docente entre otras.

\section{DELIMITACIÓN DEL PROBLEMA}

En el Instituto Tecnológico de Lázaro Cárdenas Michoacán, en el 2009, inicio la carrera de Ingeniería en Gestión Empresarial con una población estudiantil de 95 alumnos de los cuales a la fecha han egresado de la primera generación un total de 81 profesionistas de este perfil académico y debido a la escasa vinculación escuela-empresa y a la falta de personal no se ha dado seguimiento a los mismos por parte del departamento de Gestión tecnológica y 
Vinculación encargado de realizar esta actividad, por lo que se desconoce qué porcentaje de los egresados están empleados, que actividades están desempeñando, cuáles son sus requerimientos y expectativas, si el perfil de egreso es acorde a las demandas de las empresas empleadoras, así como los requerimientos que valora el mercado laboral en cuanto a conocimientos, habilidades y valores desarrollados en el instituto.

Este problema es realmente preocupante y plantea la necesidad de llenar este vacío ya que es de vital importancia conocer el nivel de empleabilidad y los requerimientos del mercado laboral en cuanto a competencias profesionales se requieren de los egresados. Por lo que la presente investigación es un primer acercamiento al seguimiento de los alumnos egresados de este programa académico. Razón por lo cual se plantea la siguiente interrogante ¿Las competencias profesionales tienen una relación directa positiva en la empleabilidad de los Ingenieros en Gestión Empresarial egresados del ITLAC?

\section{JUSTIFICACIÓN}

El mercado laboral requiere en la actualidad profesionistas competentes que cuenten con los conocimientos, habilidades y valores que den respuesta a los requerimientos que este sector demanda en el Instituto Tecnológico de Lázaro Cárdenas con el propósito de hacer frente a las circunstancias, exigencias y situaciones que se presentan en forma diaria en los diferentes ambientes de trabajo surge la necesidad de realizar una revisión constante de los conocimientos, destrezas y valores de los profesionistas de Ingeniería en gestión Empresarial y una potenciación de sus capacidades y competencias clave tendientes a incrementar la empleabilidad y disminuir el desempleo.

El desarrollo de competencias para la empleabilidad por medio de la educación deben de ayudar, por un lado, a potenciar la creatividad y la iniciativa para generar una cultura emprendedora, y por otro lado, desarrollar las capacidades y potencialidades humanas y necesarias en los egresados de este perfil profesiona, lo cual permitel cerrar la brecha entre los conocimientos teóricos, las habilidades y las destrezas requeridas por los empresarios y por las nuevas tendencias de la economía del país y del mundo, logrando que de esta manera se incremente la empleabilidad de los egresados de Ingeniría en Gestion Empresarial. Lo anterior genera crecimiento y desarrollo en el país y por consiguiente eleva el nivel de ingresos de los profesionales de la gestion empresarial y les brinda oportunidades en todos los campos de la vida de cada egresado. 
En esta investigación se definen las competencias profesionales como: las actuaciones integrales para identificar, interpretar, argumentar y resolver problemas con integridad y compromiso ético, movilizando los diferentes saberes: ser, hacer y conocer Tobón (2010).

\section{HIPÓTESIS}

Las competencias profesionales tienen una relación directa positiva en la empleabilidad de los Ingenieros en Gestión Empresarial egresados del ITLAC.

\section{MARCO TEÓRICO CONCEPTUAL}

Etimológicamente, el concepto de competencia proviene del latín competentia y dentro de las acepciones que le otorga la Real Academia Española se encuentran: incumbencia, pericia, aptitud, idoneidad para hacer algo o intervenir en un asunto determinado, oposición o rivalidad entre dos o más que aspiran a obtener la misma cosa o situación de empresas que rivalizan en un mercado ofreciendo o demandando un mismo producto o servicio. En consecuencia se señalan tres significados: competencias como rivalidad o contienda, como incumbencia y como aptitud e idoneidad (RAE, 1999).

En ocasiones se defiende la postura de que competencia laboral engloba el concepto de competencia profesional, porque lo laboral implica todo lo relacionado con el mundo del trabajo, ya sea profesión u oficio. En la literatura se habla indistintamente de competencias profesionales y competencias laborales. Las definiciones son mayoritariamente comunes pero separadas por el mero concepto semántico. Según la postura de diferentes autores y organismos nacionales e internacionales las conceptualizan de la siguiente forma:

\subsection{COMPETENCIAS PROFESIONALES}

"Las competencias profesionales son el resultado de la integración, esencial y generalizada de un complejo conjunto de conocimientos, habilidades, y valores profesionales que se manifiestan a través de un desempeño profesional eficiente en la solución de los problemas de su profesión pudiendo incluso resolver aquellos no predeterminados" (Forgas, 2003:112). Capacidades para hacer algo de modo idóneo que resultan de un proceso complejo de asimilación integrativa por parte del aprendiz de saberes conceptuales, saberes procedimentales y actitudes que se lleva cabo en la fase de ejercitación dentro del proceso enseñanza aprendizaje (Díaz-Barriga, 2009). 


\section{PROPUESTA TEÓRICO METODOLÓGICA PARA EL ANÁLISIS DE LAS COMPETENCIAS \\ PROFESIONALES Y SU IMPACTO EN LA EMPLEABILIDAD \\ DOI: http://dx.doi.org/10.5007/1983-4535.2015v8n1p110}

Por su parte la Organización Internacional del Trabajo (OIT, 1993:37) define el concepto de "competencias profesionales como la idoneidad para realizar una tarea o desempeñar un puesto de trabajo eficazmente por poseer las calificaciones requeridas para ello. En este caso, los conceptos de competencia y calificación, se asociación fuertemente dado que la calificación se considera una capacidad adquirida para realizar un trabajo o desempeñar un puesto de trabajo".

Las competencias profesionales definen el ejercicio eficaz de las capacidades que permiten el desempeño de una ocupación, respecto a los niveles requeridos en el empleo. Es algo más que el conocimiento técnico que hace referencia al saber y al saber- hacer. El concepto de competencia engloba no solo las capacidades requeridas para el ejercicio de una actividad profesional, sino también un conjunto de comportamientos, facultad de análisis, toma de decisiones, transmisión de información considerados necesarios para el pleno desempeño de la ocupación. Instituto Nacional de Empleo en España (INEM: 1995:21).

Las competencias profesionales son un conjunto de conocimientos, habilidades, destrezas y actitudes adquiridos a través de procesos formativos o de la experiencia laboral, que permiten desempeñar y realizar roles y situaciones de trabajo requeridas en el empleo (R. decreto 676/1993). Sin embargo la OCDE (2005) las conceptualiza como: la capacidad para responder exitosamente a una demanda, tarea o problemas complejos movilizando y combinando recursos personales (cognitivos y no cognitivos) y del entorno.

Las competencias profesionales: son actuaciones integrales para identificar, interpretar, argumentar y resolver problemas con integridad y compromiso ético, movilizando los diferentes saberes: ser, hacer y conocer (Tobón, 2010). (Vargas, 2001) destaca que las competencias son características permanentes de las personas, se ponen de manifiesto cuando se ejecuta una tarea o un trabajo no están asociadas con el éxito sino que se asume que realmente lo causan, pueden ser generalizadas a más de una actividad y combinan lo cognoscitivo, lo afectivo y lo conductual.

\subsection{COMPETENCIAS LABORALES}

"Competencia laboral es la aptitud de un individuo para desempeñar una misma función productiva en diferentes contextos y con base en los requerimientos de calidad esperados por el sector productivo. Esta aptitud se logra con la adquisición y desarrollo de conocimientos, habilidades y capacidades que son expresados en el saber, el hacer y el saber 
hacer" (Mertens, 2000: 12). Se conciben como una compleja estructura de atributos necesarios para el desempeño de situaciones específicas. Es una compleja combinación de atributos (conocimientos, actitudes, habilidades y valores) y las tareas que se tienen que desempeñar en determinadas situaciones. Que se ponen en acción para un desempeño adecuado en un contexto y la cultura del lugar de trabajo. Nos permite integrar la ética y valores como elementos del desempeño competente". Irigoin y Vargas (2002:47).

Competencia laboral: es la capacidad subyacente en una persona que esta causalmente relacionada con el desempeño, referido a un criterio superior o efectivo en un trabajo o situación (Spencer \& Spencer, 1993). Por su parte (McClellan, 1973) define las competencias laborales como: características de un individuo que guardan una relación causal con el desempeño efectivo o superior en el puesto- motivos, características de personalidad, habilidades, aspectos de autoimagen o un conjunto de conocimientos que un individuo está usando. No obstante (Levy-Leboyer, 1997: 13) define las competencias laborales como: "Repertorios de conocimientos que algunos dominan mejor que otros, lo que les hace eficaces en una situación determinada".

Posee competencia laboral (Agudelo, 2002:23) "quien dispone de los conocimientos, habilidades, aptitudes y actitudes necesarios para desempeñarse eficazmente en situaciones específicas de trabajo", lo cual le permite resolver, los problemas que se le presenten en el ejercicio de sus funciones, en forma autónoma y flexible, y está capacitado para colaborar en su entorno profesional y en la organización de su trabajo. En este sentido la competencia sería una capacidad genéticamente determinada que se expresa a través del lenguaje y el desempeño estaría dado por el uso efectivo de esta capacidad en situaciones concretas (Chomsky, 1965).

Capacidad de actuar eficazmente en un número determinado de situaciones, capacidad basada en los conocimientos, pero que no se limita a ellos (Perrenoud, 1999). Capacidad o potencia para actuar de manera eficaz en un contexto determinado (Eurydice, 2002). Habilidades vinculadas con el desempeño autónomo, el conocimiento aplicado y aplicable, el conocimiento en acción, el saber resultante del saber hacer y saber explicar lo que se hace. Brailovsky y Acosta, (2006). Repertorio de estrategias coordinadas para resolver una demanda especifica correspondiente a un contexto habitual (educativo, familiar, profesional, personal de la actividad humana (Monereo, 2003). 


\section{PROPUESTA TEÓRICO METODOLÓGICA PARA EL ANÁLISIS DE LAS COMPETENCIAS \\ PROFESIONALES Y SU IMPACTO EN LA EMPLEABILIDAD \\ DOI: http://dx.doi.org/10.5007/1983-4535.2015v8n1p110}

Competencias laborales son el "conjunto integrado de conocimientos, habilidades, actitudes y valores así como rasgos de personalidad y motivaciones innatas o subyacentes en una persona (ver figura 1) que le predisponen para desempeñar con éxito, los requisitos y exigencias de un puesto de trabajo, ocupación, cometido o papel en un contexto profesional dado" (Lombana, 1979:163).

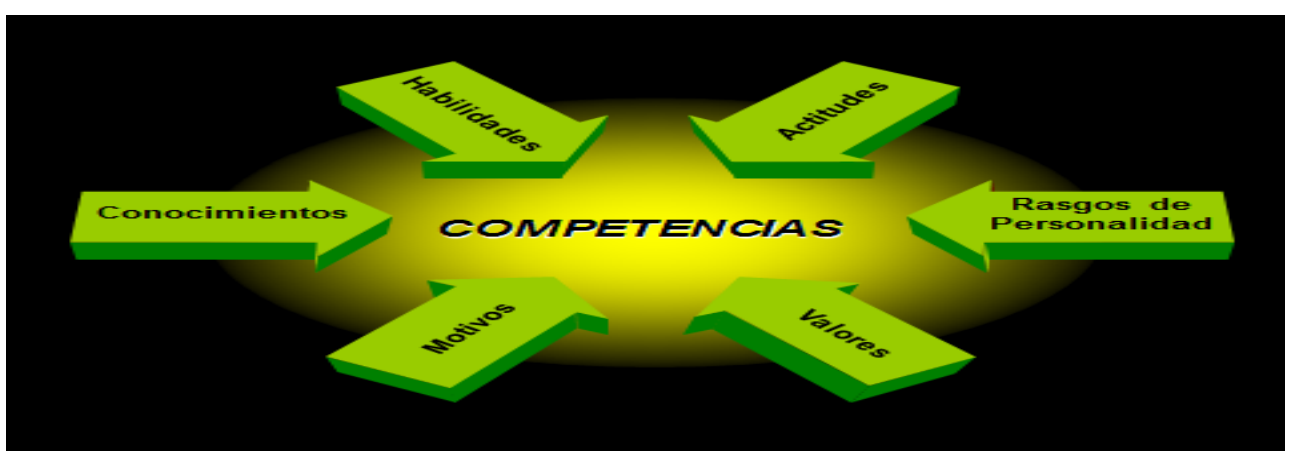

Figura 1 Componentes de las competencias Fuente: Elaboración propia.

Las competencias presentes en una persona, son las que permiten un desempeño diferenciador o exitoso, no todas las personas en su desempeño podrían ser exitosas por el solo hecho de desearlo, es necesario tener esa cualidad personal que le permite realizar una actividad en forma más exitosa que otra persona. Es individual en cuanto a que cada persona tiene sus propias competencias, no son copiables e imitables y son medibles en cuanto son identificables a través de instrumentos de medición confiables científicamente.

Las competencias, además, son observables en la realidad del trabajo, e igualmente en situaciones de evaluación, y ponen en práctica de manera integrada aptitudes, rasgos de personalidad y conocimientos. Por lo tanto, las considera un lazo de unión entre las características individuales y las cualidades requeridas para realizar las actividades del puesto. (Ver figura No. 2).

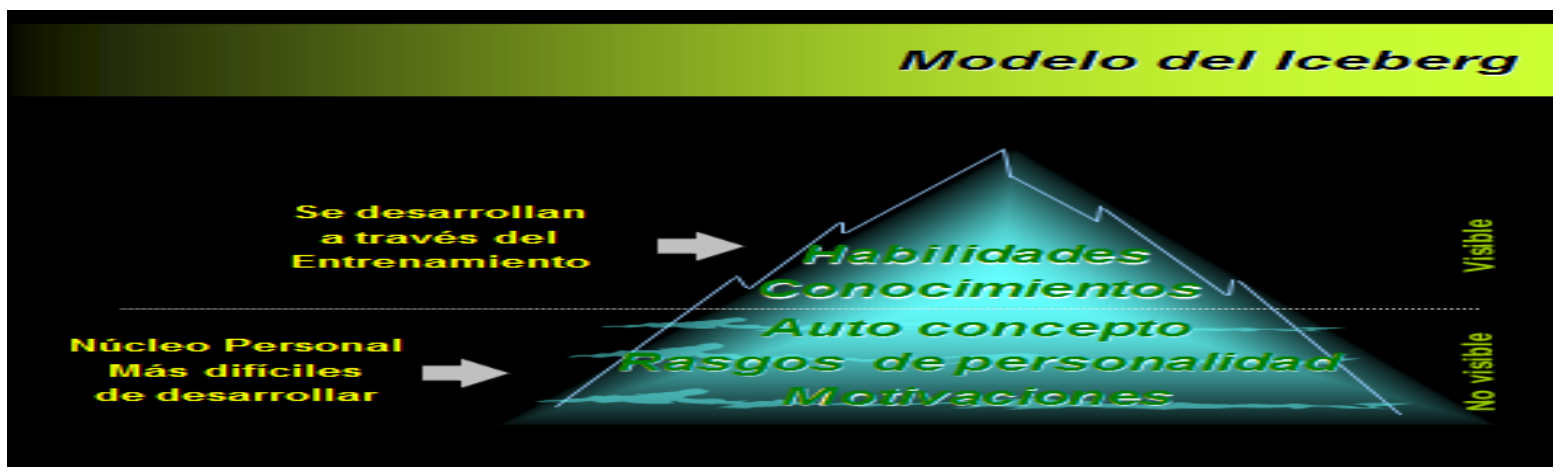

Figura 2 Modelo del Iceberg en el desarrollo de competencias.

Fuente: elaboración propia en base a Spencer \& Spencer (1993) 
Habilidades: Capacidad de una persona para hacer algo. Conocimientos: La información que una persona tiene de un área particular. Auto concepto: Lo que cada persona considera que es, con respecto a sus valores, características, actitudes e imagen. Ejemplo: autoconfianza al propio desempeño. Rasgos de personalidad: Aspecto típico del comportamiento de una persona. Características físicas y respuestas consistentes a situaciones o información. Ejemplo: Resolver problemas bajo estrés. Motivaciones: Aquellas cosas que una persona considera o desea consistentemente. Las motivaciones dirigen, conllevan y seleccionan el comportamiento hacia ciertas acciones y objetivos y lo alejan de otros.

\subsection{EMPLEABILIDAD}

La palabra empleabilidad no existe como tal en la lengua castellana surgió de la palabra inglesa "employability", que proviene de la unión de la palabra: “employ" (empleo) y "hability" (habilidad) ya unidas formaron: employability, la cual se ha adaptado sin mayores cambios al español como "empleabilidad". Habilidad para obtener y conservar un empleo y evitar el desempleo Campos (2003). Ser empleable significa tener la habilidad de poseer un empleo, y no ser empleable significa lo contrario, Rentería (2004).

La empleabilidad, por tanto, está relacionada con el desarrollo de competencias que son valoradas en el mercado de trabajo, es decir, se asume la existencia de una serie de competencias que mejoran las posibilidades de los individuos que las poseen de encontrar un empleo y de mantenerlo en el tiempo, por lo que se presentan algunas definiciones relevantes de empleabilidad que contribuyen a establecer la dimensión de esta variable. Con este objetivo se ha efectuado una revisión y análisis de la literatura científica presentando la siguiente tabla a modo de resumen.

Tabla 1 Definiciones de empleabilidad

\begin{tabular}{|l|l|}
\hline AUTORES/REFERENCIA & \multicolumn{1}{c|}{ DEFINICIÓN DE EMPLEABILIDAD } \\
\hline Martínez y Renteria (2006) & La capacidad de obtener un empleo y mantenerse en este. \\
\hline $\begin{array}{l}\text { Silla, Gracia y Peiro, } \\
\text { 2005:99) }\end{array}$ & "Capacidad para encontrar otro trabajo en caso de perder o abandonar el actual" \\
\hline OIT (2004: 49) & $\begin{array}{l}\text { "las competencias y cualificaciones transferibles que refuerzan la capacidad de las personas } \\
\text { para aprovechar las oportunidades de educación y de formación que se les presenten con } \\
\text { miras a encontrar y conservar un trabajo decente, progresar en la empresa o al cambiar de } \\
\text { empleo y adaptarse a la evolución de la tecnología y de las condiciones del mercado de } \\
\text { trabajo" }\end{array}$ \\
\hline York, M. (2004) & $\begin{array}{l}\text { Conjunto de logros, habilidades-conocimientos y atributos personales que aumentan la } \\
\text { probabilidad de los graduados de encontrar un empleo y de tener éxito en sus puestos, lo cual } \\
\text { les beneficia a ellos mismos, a la fuerza de trabajo, a la comunidad y a la economía. }\end{array}$ \\
\hline Campos, G. (2003) & Habilidad para obtener o conservar un empleo y para evitar el desempleo. \\
\hline Van der Heijden, B. & "Capacidad de ser empleado en un trabajo" \\
\hline
\end{tabular}




\begin{tabular}{|l|l|}
\hline (2002: 44) & \\
\hline Thijssen, J.(2000: 9) & $\begin{array}{l}\text { "Capacidad individual para desempeñar una variedad de funciones en un mercado laboral } \\
\text { dado" }\end{array}$ \\
\hline $\begin{array}{l}\text { Neffa, J.C., Panigo, D. y } \\
\text { Pérez, P. (2000: 27). }\end{array}$ & $\begin{array}{l}\text { "La probabilidad que tienen las personas desocupadas de encontrar empleo en un período } \\
\text { dado". }\end{array}$ \\
\hline $\begin{array}{l}\text { Groot y Maassen van den } \\
\text { Brink (2000: 574) }\end{array}$ & $\begin{array}{l}\text { "El número de tareas que pueden asignarse a un trabajador o la cantidad de asistencia } \\
\text { necesaria en el trabajo. }\end{array}$ \\
\hline FUNDIPE (1999) & $\begin{array}{l}\text { Es la capacidad que una persona tiene para tener un empleo que satisfaga sus necesidades } \\
\text { profesionales, económicas, de promoción y de desarrollo a lo largo de su vida. }\end{array}$ \\
\hline $\begin{array}{l}\text { Hillage, J. and Pollard, E. } \\
\text { (1998:15). }\end{array}$ & $\begin{array}{l}\text { "Capacidad de los individuos para obtener un empleo inicial, mantener el empleo, moverse } \\
\text { entre roles dentro de una misma organización, obtener un empleo nuevo si se requiere, e } \\
\text { idealmente asegurar un trabajo adecuado y suficientemente satisfactorio" }\end{array}$ \\
\hline
\end{tabular}

Fuente: Elaboración propia.

La esencia de la empleabilidad está en la capacidad para encontrar y mantener un trabajo, en cuyo caso los elementos claves en este proceso serán la preparación, las actitudes, la iniciativa, flexibilidad y disposición del trabajador. La empleabilidad deja de manifiesto la responsabilidad del propio sujeto para emplearse y permanecer en el mercado laboral. Por lo que la empleabilidad como competencia sugiere que una vez conseguido el empleo, este cumpla con las expectativas de la persona trabajadora, satisfaciendo sus necesidades, profesionales, económicas, de promoción y de desarrollo por lo que se requiere la creación de más empleos y de mejor calidad.

La oportunidad de trabajo tiene un impacto positivo en la empleabilidad puesto que la ocupación constituye una base privilegiada para recoger las experiencias y asumir las actitudes y la mentalidad que la empleabilidad demanda. Lo anterior señala la necesidad de que el trabajador tenga la capacidad de poder desempeñarse en diferentes actividades laborales. Aspecto de gran relevancia en el competitivo mercado laboral que caracteriza al México de hoy incorporado a la globalización, que obliga a los profesionales a desarrollar capacidades que le permitan desempeñar de forma flexible una variedad de funciones

Las personas podrían ser más empleables cuando han adquirido una educación y una formación de base amplia y calificaciones básicas y transferibles de alto nivel, incluidos el trabajo en equipo, la capacidad para resolver problemas, las tecnologías de la comunicación e información, el conocimiento de idiomas, la capacidad de aprender a aprender, así como competencias para protegerse a sí mismos como a sus compañeros contra los riesgos y las enfermedades profesionales.

Dentro de estos factores se pueden citar los factores individuales relativos a las etapas de la vida de las personas; así como los factores educativos (enseñanzas básicas, lenguaje, matemáticas), laborales académicos y del entorno. No obstante la persona es la protagonista de su empleabilidad. Y si no quiere o no asume la conveniencia de estar al día y la ambición 
de mejora así como los sacrificios en un esfuerzo de aprendizaje, una mayor flexibilidad o más movilidad funcional o geográfica, poco podrá hacerse para mejorar su empleabilidad. Las cuestiones vinculadas a la empleabilidad no dejan al margen la influencia de los empleadores, ya que finalmente son ellos quienes deciden la aceptación o el rechazo de los candidatos a un puesto de trabajo. Esta es una de las razones por las cuales la empleabilidad no puede ser abordada desde una perspectiva única pues es construida a partir de múltiples actores sociales.

Tabla 2 Actores sociales que contribuyen a la empleabilidad

\begin{tabular}{|c|c|c|}
\hline $\begin{array}{l}\text { ETAPAS DE LA } \\
\text { VIDA }\end{array}$ & $\begin{array}{l}\text { INSTITUCIONES/SUJETOS } \\
\text { INVOLUCRADOS }\end{array}$ & POSIBLES MEDIDAS Y ACCIONES A DESARROLLAR \\
\hline INFANCIA & FAMILIA/ESCUELA & Testimonial, deporte, valores y educación relacional. \\
\hline JUVENTUD & $\begin{array}{l}\text { ESCUELA/CENTROS } \\
\text { DE FORMACIÓN DE } \\
\text { FAMILIA }\end{array}$ & $\begin{array}{l}\text { Fomento del deporte } \\
\text { Educación con cierto propósito } \\
\text { Desarrollo de conceptos: sentido de utilidad, sentido de eficacia, } \\
\text { trabajo bien hecho. } \\
\text { Orientación sobre carreras profesionales. } \\
\text { Visión a largo plazo y planes para alcanzarla. }\end{array}$ \\
\hline $\begin{array}{l}\text { SI } \\
\text { ESTUDIOS } \\
\text { SUPERIORES }\end{array}$ & $\begin{array}{l}\text { CENTROS } \\
\text { UNIVERSITARIOS }\end{array}$ & $\begin{array}{l}\text { Mayor participación de profesionales y personas de trabajo. } \\
\text { Fomento de prácticas. } \\
\text { Orientación para el diseño de la propia carrera. } \\
\text { Orientación hacia el empleo. }\end{array}$ \\
\hline EMPLEADO & EMPRESA/SINDICATO & $\begin{array}{l}\text { En un clima hacia la consecución de objetivos y resultados, } \\
\text { creando un clima de aprendizaje. } \\
\text { Fomento de practicas } \\
\text { Orientación hacia el propio diseño de la carrera. } \\
\text { Organizando sistemáticos cambios que supongan el desarrollo de } \\
\text { la carrera. } \\
\text { Entrevista de evaluación y de consejo. }\end{array}$ \\
\hline $\begin{array}{l}\text { EMPLEADO } \\
\text { ADULTO }\end{array}$ & $\begin{array}{l}\text { SINDICATO/ } \\
\text { EMPRESA } \\
\text { ORGANISMOS } \\
\text { PÚBLICOS } \\
\end{array}$ & $\begin{array}{l}\text { Formación profesional } \\
\text { Ayuda para la búsqueda del empleo desde el empleo actual. } \\
\text { Formación actualizada. }\end{array}$ \\
\hline $\begin{array}{l}\text { DESEMPLEA } \\
\text { DO } \\
\text { ADULTO }\end{array}$ & $\begin{array}{l}\text { SERVICIOS } \\
\text { DESEMPLEO } \\
\text { (INAPAM) }\end{array}$ & $\begin{array}{l}\text { Formación, bolsa de trabajo, ofertas de empleo, agencias de } \\
\text { colocación e integración de actividades sociales }\end{array}$ \\
\hline JUBILADO & $\begin{array}{l}\text { Ayuntamientos, } \\
\text { Organismos públicos y } \\
\text { privados, ONG'S }\end{array}$ & $\begin{array}{l}\text { Formación, colaboración con instituciones, voluntariado e } \\
\text { Integración en actividades sociales }\end{array}$ \\
\hline
\end{tabular}

Fuente: elaboración propia en base a FUNDIPÉ (1999)

\section{MARCO CONTEXTUAL}

El Sistema Nacional de Educación Superior Tecnológica (SNEST), cuyo antecedente es el Sistema Nacional de Institutos tecnológicos de la Secretaria de Educación Pública (SEP) históricamente inicio en el campo de la educación y la investigación con la fundación de los Institutos Tecnológicos Regionales de Durango y Chihuahua en 1948, como un proyecto derivado del Instituto Politécnico Nacional (IPN). Casi cuatro décadas después a finales de 
los años ochenta, se crearon los primeros Institutos Tecnológicos Descentralizados, instituciones de educación superior tecnológica con personalidad jurídica y patrimonio propios (Organismos Públicos Descentralizados de Carácter Estatal), y en cuya creación y órgano de gobierno participaron los tres niveles de gobierno, así como representantes de los sectores de la sociedad.

En la actualidad el Sistema nacional de educación Superior Tecnológica (SNEST) cuenta con 261 instituciones en el país (ver figura), de las cuales 130 son institutos tecnológicos descentralizados, 125 son institutos tecnológicos federales, cuatro centros regionales de optimización y desarrollo de equipo (CRODE), y dos centros de investigación: el Centro Nacional y Desarrollo Tecnológico (CENIDET) y el centro Interdisciplinario de Investigación y Docencia en Educación Técnica (CIIDET). Con una matrícula estudiantil de 491,165 estudiantes de licenciatura y posgrado en todo el territorio nacional incluido el distrito federal.

El 23 de Julio del 2014 (D.O.F.) deja de existir la Dirección general de Educación Superior tecnológica de la SEP, para dar lugar a la creación de un órgano desconcentrado denominado TECNOLÓGICO NACIONAL DE MÉXICO, donde la conformación de su cobertura es la siguiente:

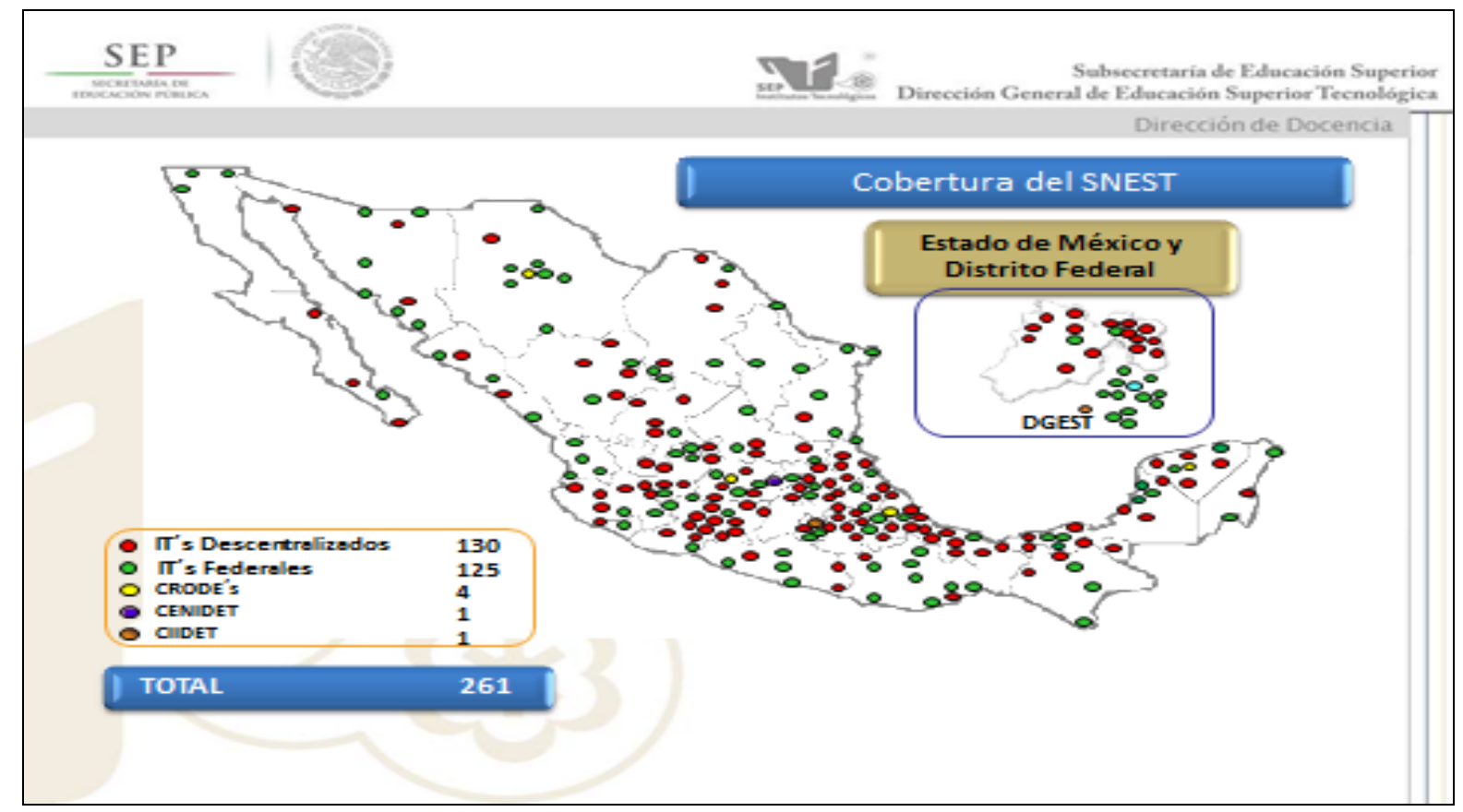

Figura 3 Cobertura del Sistema Nacional de Educación Superior en México. Fuente: Acosta-González, (2011). 
La oferta educativa del Instituto Tecnológico de Lázaro Cárdenas, consistente en 06 carreras de ingeniería: Sistemas Computacionales, Electrónica, Electromecánica, Química, Industrial e Ingeniería en Gestión Empresarial, 02 carreras del área económico-administrativa: Administración y Contaduría; hacen necesario que los 107 docentes que forman nuestra plantilla docente se encuentren distribuidos en los diversos departamentos académicos de nuestra estructura orgánica en función del perfil profesional de la licenciatura de la cual egresaron. La matrícula estudiantil de estos programas académicos durante el periodo agosto diciembre del 2014 es la siguiente:

\begin{tabular}{lccccc}
\multicolumn{1}{c}{ CARRERA } & NUEVO INGRESO & \multicolumn{2}{c}{ REINGRESO } & TOTAL \\
Ingeniería en Sistem as Computacionales & HOMBRES & MUJERES & HOMBRES & MUJERES & \\
Ingeniería Electromecánica & 68 & 30 & 181 & 81 & 360 \\
Ingeniería Electrónica & 86 & 5 & 179 & 8 & 278 \\
Ingeniería Química & 94 & 4 & 212 & 15 & 325 \\
Ingeniería Industrial & 31 & 34 & 94 & 93 & 252 \\
Ingeniería en Gestión Empresarial & 69 & 35 & 189 & 112 & 405 \\
Licenciatura en Administración & 39 & 71 & 65 & 157 & 332 \\
Licenciatura en Administración S.A. & 18 & 38 & 97 & 132 & 285 \\
Licenciatura en Contaduría & 35 & 55 & 70 & 114 & 274 \\
Contador Público & 0 & 0 & 19 & 37 & 56 \\
\multicolumn{1}{c}{ TOTAL } & 22 & 29 & 21 & 41 & 113 \\
\hline
\end{tabular}

Cuadro 1 Matricula estudiantil por programa académico en el ITLAC.

Fuente: elaboración propia en base a información proporcionada por el departamento de servicios escolares del instituto.

El Tecnológico No. 56 (Nombre inicial del Instituto Tecnológico de Lázaro Cárdenas), se creó como una extensión del Tecnológico de Morelia a partir de las gestiones realizadas por el Patronato Pro-fundación del Centro de Estudios Superiores de Lázaro Cárdenas, iniciando sus actividades el día 13 de octubre de 1987. Ofreciendo las Licenciaturas en Ingeniería Química e Ingeniería Electromecánica; con una población de 77 estudiantes en turno vespertino, hizo uso de las instalaciones del Centro de Estudios Tecnológicos del Mar No. 16, ubicado en la Isla del Cayacal facilitadas por el Ing. Marco Antonio Peña, teniendo como Coordinador al Ing. Jorge Aguilar Piñón.

Desde entonces nuestro plantel ha ido creciendo y mejorando constantemente para cubrir las expectativas de los alumnos y que la industria y la sociedad exige. Contando con un total de siete directores que han estado a cargo de este Instituto Tecnológico, siendo actualmente el Ing. José Ángel Esquivel Tovar. En este momento el Instituto Tecnológico de Lázaro Cárdenas, se sitúa sobre una superficie de 26.60-27.01 hectáreas y se encuentra 
ubicado entre las Avenidas: Melchor Ocampo, Tariácuri y Narciso Bassols, el libramiento en la Ciudad de Lázaro Cárdenas, Michoacán y limita con el Ejido la florida y el ejido de Guacamayas, además se localiza a $695 \mathrm{Km}$. de la Ciudad de México (vía corta).

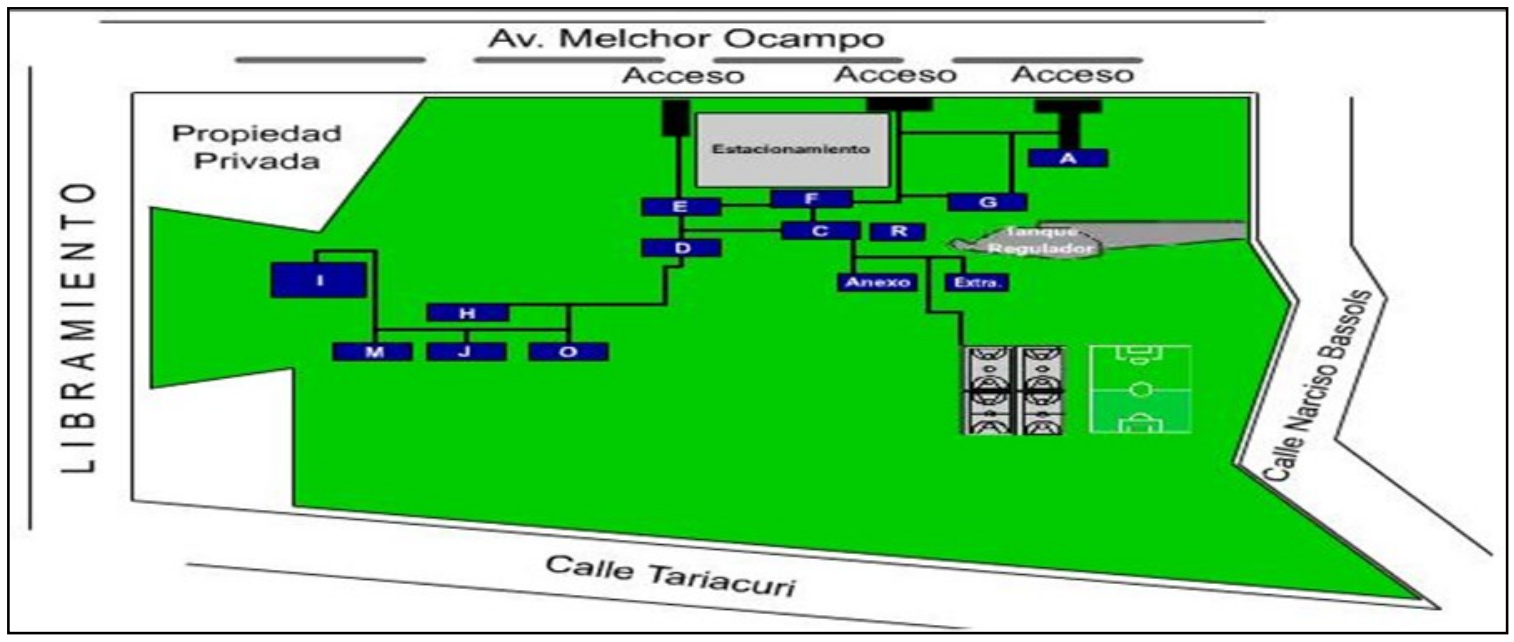

Figura 4 Croquis de Localización del ITLAC.

Fuente: Elaboración propia en base a información proporcionada por el departamento de planeación del ITLAC

La distribución de la matrícula estudiantil en el periodo agosto diciembre del 2014 está conformada de la siguiente forma:

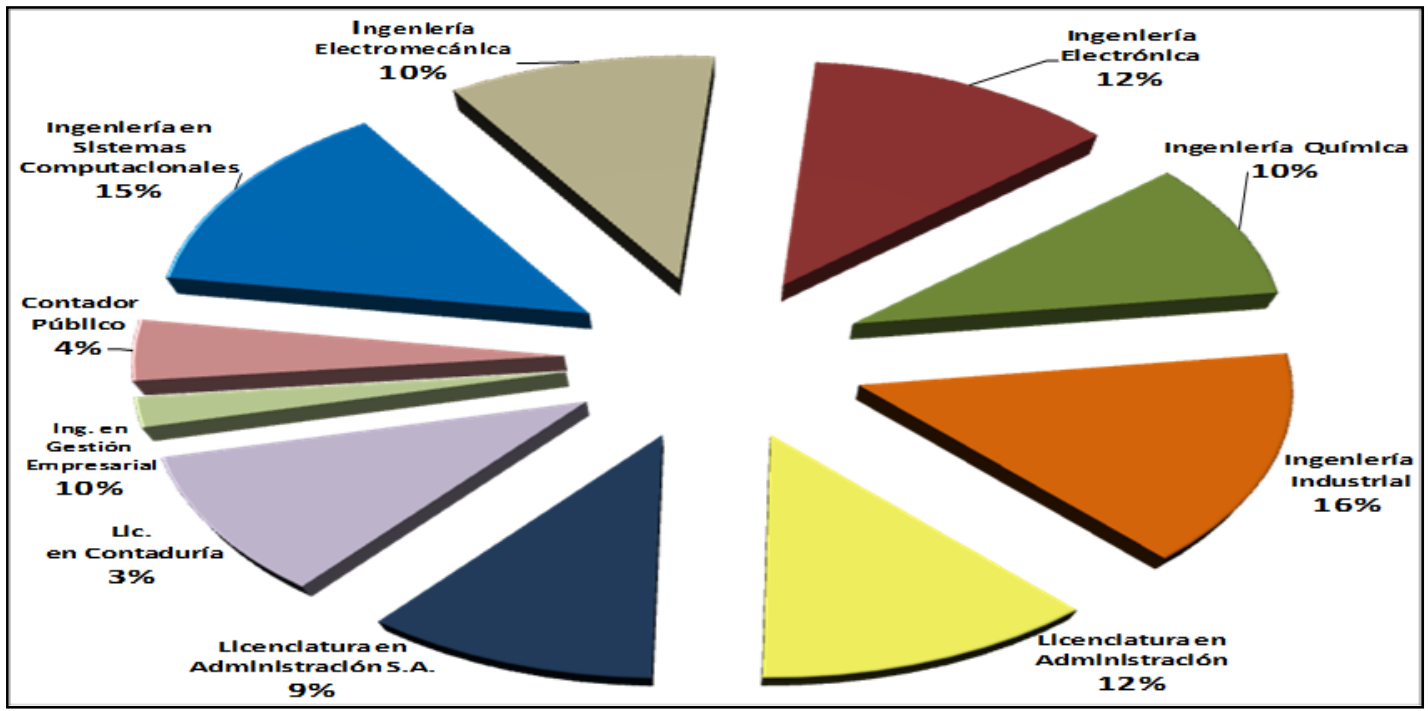

Grafico 1 Distribución de matrícula estudiantil por programa académico.

Fuente: Elaboración propia en base a datos proporcionados por el departamento de servicios escolares del instituto. 


\section{MÉTODO}

En la presente investigación se emplea el método mixto para obtener una comprensión y análisis más completo de las variables objeto de estudio (ver cuadro 2), por un lado se mide cada variable presuntamente relacionada y por el otro se analiza como es y cómo se manifiesta la percepción de los egresados de Ingeniería en Gestión Empresarial y los empleadores de los mismos en la ciudad de Lázaro Cárdenas Michoacán, el cual basado en la revisión literaria, partiendo de la conceptualización de ambas se analizan datos presentados por estudiosos e investigadores profesionales y organismos nacionales e internacionales, que explican el fenómeno objeto de estudio, los cuales demuestran la incidencia de una influencia positiva de las competencias profesionales en la empleabilidad de los egresados de este perfil profesional.

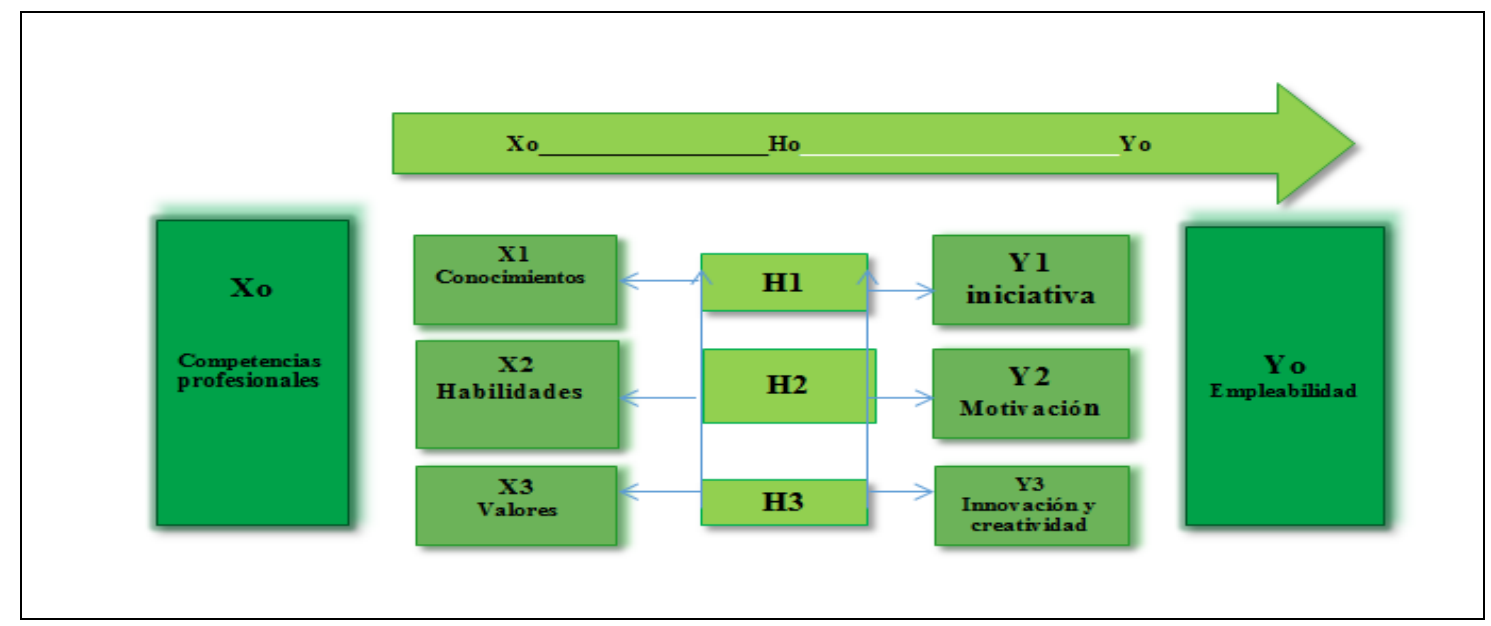

Cuadro 2 Despliegue de variables, dimensiones e hipótesis.

Fuente: Elaboración propia.

\section{PROPUESTA DE INVESTIGACIÓN}

Una vez que se realizó la revisión literaria para efectos de esta investigación. Las competencias profesionales se definen como: las actuaciones integrales para identificar, interpretar, argumentar y resolver problemas con integridad y compromiso ético, movilizando los diferentes saberes: ser, hacer y conocer Tobón (2010).

El marco teórico propuesto es el modelo de emprendimiento en red González y Gálvez (2008) el cual da la oportunidad al Instituto Tecnológico de Lázaro Cárdenas Michoacán de responder a los retos impuestos por la sociedad que hace necesaria la propuesta de nuevos modelos de enseñanza que no se limiten a la formación de empleados, 
sino que empiecen a formar profesionales emprendedores que inicien y creen sus propias empresas. Didrikson, Arteaga, y Campos (2004), con el propósito fundamental de enmarcar el autoempleo como una alternativa de solución real al desempleo.

Se presenta a los estudiantes de la carrera de Ingeniería en gestión Empresarial del ITLAC como un potencial emprendedor cuyas habilidades deben de ser desarrolladas y aplicadas durante sus estudios universitarios en la institución educativa, para que generen un negocio propio y por ende contrarrestar el problema generalizado del desempleo que se vive en nuestro país considerando que ya cuentan con los conocimientos requeridos a su perfil profesional de egreso.

Los emprendedores son la parte creativa e innovadora, no solamente en la creación de empresas, sino en la creación de nuevas formas de producción, administración y generación de productos. La verdadera función del emprendedor es tomar iniciativas e innovar, el rol del dirigente es actuar sobre la motivación Schumpeter (1984). La cultura empresarial proporciona el marco de referencia de valores que moldean las actividades productivas y utilitarias y es la que proporciona el esquema valorativo que establece el sentido de un sistema de relaciones entre las características de las organizaciones llamadas empresas y la de sus principales actores, empresarios y administradores. Vargas (2007).

Tabla $\mathbf{n}^{\mathbf{0}} . \mathbf{3}$ Metodología para el modelo de emprendimiento en red

\begin{tabular}{|c|c|}
\hline Etapa 0 & $\begin{array}{l}\text { Conocimiento de las redes empresariales: en esta etapa, el emprendedor conoce generalidades } \\
\text { como su razón de ser, el funcionamiento, las ventajas y las responsabilidades del trabajo en red. } \\
\text { El producto de esta etapa es un emprendedor sensibilizado respecto al tema, susceptible de } \\
\text { desarrollar la visión de crear una empresa para ingresar en una red empresarial. }\end{array}$ \\
\hline Etapa 1 & $\begin{array}{l}\text { Adquisición de la motivación e idea de negocio: en esta etapa el emprendedor busca los } \\
\text { estímulos y los objetivos para crear un negocio (Mitton, 1989). Cuando contempla la creación } \\
\text { de una empresa dentro de una red, el emprendedor debe analizar las motivaciones personales } \\
\text { que puede tener para vincularse a ella, a un escenario donde las relaciones, no solo } \\
\text { comerciales, sino fundamentalmente personales, y donde se busca construir lazos de } \\
\text { confianza, solidaridad y cooperación. Si el emprendedor no tiene competencias personales para } \\
\text { trabajar en equipo y para solucionar conflictos, ni le interesa desarrollarlas, puede en esta etapa } \\
\text { descubrir que es insuficiente su motivación. Para crear una empresa sostenible en una red y } \\
\text { desistirá de ello. Pero si, por el contrario, cuenta con la motivación suficiente seguirá adelante } \\
\text { en su empeño. }\end{array}$ \\
\hline Etapa 2 & $\begin{array}{l}\text { Validación de la idea: esta etapa es quizás la más importante. Le sirve al emprendedor para } \\
\text { evaluar las diferentes ideas de negocio y descartar algunas que sean imposibles de lograr Gibb } \\
\text { (1988) propone una serie de preguntas que pueden ayudar en esta tarea ¿Cuál es exactamente la } \\
\text { idea? ¿Funcionaria? ¿Está claramente identificada la necesidad que ha de satisfacer? ¿es legal? } \\
\text { ¿es posible entrar en el mercado? Etc. }\end{array}$ \\
\hline Etapa 3 & $\begin{array}{l}\text { Identificación de los recursos necesarios: en esta etapa el emprendedor debe de identificar } \\
\text { detalladamente los recursos necesarios, el tiempo, la calidad de sus proveedores y materiales, } \\
\text { los consumidores y la escala de negocio en el caso de la creación de una empresa } \\
\text { independiente y aislada. En el caso de una empresa en red, el emprendedor debe de tener muy } \\
\text { claro cómo está compuesta esta, como funciona, como son sus relaciones, cuáles son sus }\end{array}$ \\
\hline
\end{tabular}




\begin{tabular}{|c|l|}
\hline & $\begin{array}{l}\text { productos principales, tecnología, equipo, empleados, fortalezas y debilidades, economía } \\
\text { regional y en la generación de empleo, potencialidad para agregar valor a los productos } \\
\text { generados en la región. }\end{array}$ \\
\hline Etapa 4 & $\begin{array}{l}\text { Negociación del ingreso al mercado: en esta etapa busca crear y aplicar el plan de negocio; } \\
\text { incluyendo la negociación con bancos (capital), con empleados (mano de obra), con } \\
\text { proveedores (materia prima) y con distribuidores (canales de distribución). }\end{array}$ \\
\hline Etapa 5 & $\begin{array}{l}\text { Nacimiento o creación de la empresa: cuando la empresa es aislada depende ciento por ciento } \\
\text { del emprendedor de sus recursos y tiempo y en especial de su capital humano social (Anderson } \\
\text { y Miller, 2003). Si se trata de una red es necesario elaborar contratos para garantizarla } \\
\text { operación. }\end{array}$ \\
\hline Etapa 6 & $\begin{array}{l}\text { Supervivencia: esta etapa es la consolidación del negocio, para una empresa independiente y } \\
\text { aislada, este proceso involucra el control financiero de sus flujos, el desarrollo del mercado, la } \\
\text { estabilización del proceso de aprendizaje de sus empleados. La supervivencia de una empresa } \\
\text { en la red depende de la visión del emprendedor y de las relaciones de confianza, solidaridad y } \\
\text { cooperación que sean construidas desde la creación de la empresa. En esta etapa los miembros } \\
\text { de la red deben de realizar constantemente un análisis de solidaridad y equidad, para garantizar } \\
\text { la convivencia a largo plazo. }\end{array}$ \\
\hline
\end{tabular}

Fuente: adaptada de Gibb (1983: 53)

Elaboración propia

Para utilizar este modelo el emprendedor debe de buscar información que le permita seguir cada etapa. Quizás obtener esta información sea en algunos casos imposible porque no existe una entidad pública o privada que realice este tipo de estudios en la región o sector en que el emprendedor intenta crear su empresa. La ventaja es que en la actualidad existen entidades que intentan formar o crear redes, pues son el futuro de la región, especialmente para las pequeñas y medianas empresas.

\section{CONCLUSIONES}

El concepto de empleabilidad cobra gran relevancia en los momentos difíciles que se viven en el país donde predomina el desempleo, la crisis económica y la inseguridad. Al indagar en la literatura científica se observa la existencia de un conjunto de competencias que mejoran la probabilidad de que los egresados encuentren un empleo. Las conclusiones obtenidas son muy significativas dentro de las cuales se pueden señalar:

a) Mejorar la empleabilidad a través del desarrollo de competencias profesionales de los egresados en Ingeniería en Gestión Empresarial del ITLAC para a su vez mejorar la competitividad de la sociedad constituye un objetivo básico del sistema educativo, por lo que la empleabilidad es conceptualizada como una competencia personal.

b) Los gobernantes también son responsables de formar un marco adecuado para el aprendizaje de las competencias profesionales para la empleabilidad y para el desarrollo y el crecimiento del empleo, asumiendo normas adecuadas de contratación, atendiendo a la protección del desempleado, el plan de carrera, así como los procesos de promoción y desarrollo al interior de las empresas. 
c) La institución educativa debe garantizar la inserción y movilidad laboral de los egresados de este perfil profesional, por eso es importante realizar una revisión de propuestas curriculares, los recursos pedagógicos que ofrece e identificarlos requerimientos y demandas del mercado laboral, de tal forma que su oferta educativa sea coherente con las condiciones y necesidades del mundo contemporáneo, buscando que sus egresados cuenten con las competencias idóneas para desenvolverse en la sociedad y sean parte activa del progreso y desarrollo regional y nacional.

d) En épocas de crisis como la que actualmente se vive en el país los profesionistas egresados de este perfil profesional cuentan con la capacidad de constituir pequeñas empresas en forma personal o en red asociadas a la innovación, como instrumento a través del cual las empresas nacen, crecen y se consolidan asumiendo la gestión de las mismas los propios trabajadores. Se hace necesario potenciar este tipo de fórmulas tanto desde el sistema educativo mediante la formulación de proyectos y participando en programas de emprendedores, como desde la administración pública a través de la implementación de políticas especialmente mediante apoyos financieros o incubación de empresas desde la escuela.

\section{REFERENCIAS BIBLIOGRÁFICAS}

Acosta-Gonzalez, M.G. (2011). El proceso de diseño e innovacion curricular en competencias profesionales. DGEST. Recuperado el 13 de agosto 2013 de http://www.dest.gob.mx

Agudelo, S. (2002). Alianzas entre formación y competencia. Cinterfor/OIT. Recuperado el 12 de octubre del 2013 de:

http://www.cinterfor.org.uy/public/spanish/region/ampro/cinterfor/formacioncompetencias.ht $\underline{\mathrm{m}}$.

Chomsky, M. (1999). Aspectos de la teoría de la sintaxis. Barcelona: Gedisa.

ANUIES, (09 de septiembre 2014).Panorama de la Educación 2014.Recuperado de: http://wwwexcelsior.com.mx/nacional/2014/09/09/980761.

ANUIES, (2003). La educación superior en el siglo XXI. ANUIES. México.

Argudín, Y. (2006). Educación basada en competencias. Nociones y antecedentes. México, D.F.: Trillas. $2^{\mathrm{a}}$. Reimpresión.

Argüelles, A. (1996). Competencia laboral y educación basada en normas de competitividad. México, D.F.: Limusa.

Brailovsky, C. y Acosta, F. (2006). La formación en competencias para la gestión y la política educativa: un desafío para la educación superior en América Latina. Revista Electrónica Iberoamericana sobre Calidad, Eficacia y Cambio en Educación, No. 4, 2a . Edición pp. 2742

Campos, G. (2003). Implicaciones económicas del concepto de empleabilidad. Revista de la Facultad de Economía. BUAP, 7 (23), 12-23. 
Chomsky, M. (1999). Aspectos de la teoría de la sintaxis. Barcelona: Gedisa.

Delval, J. (2002). La escuela posible. Cómo hacer una reforma de la educación. Barcelona. España: Ariel.

Díaz-Barriga, A. (2009). Pensar la didáctica. Buenos Aires: Amorrortu.

Didrikson, A., Arteaga, C. y Campos, G. (2004). Retos y Paradigmas. El futuro de la Educación Superior en México. México: Plaza y Valdez.

D.O.F. (2014). Creación del Tecnológico Nacional de México. Decreto. 23 de julio del 2014.

EURYDICE (2002). Las competencias clave. Un concepto en expansión dentro de la educación obligatoria. Red Europea de Información en Educación Madrid. Recuperado el 12 de marzo del 2014 de:

http://eacea.ec.europea.eu/education/eurydice/thematicstudiesarchivesen.php

Forgas, J. (2003). Diseño curricular por competencias. Tesis doctoral en ciencias pedagógicas. Instituto Superior Pedagógico Frank País, Santiago de Cuba.

FUNDIPE, (1999). Informe sobre empleabilidad. Recuperado el 23 de febrero del 2014 de http://www.fundipe.es/formatos\%pdf

Gallart, M. y Jacinto, C. (1995). Competencias laborales: tema clave en la articulación educación-trabajo. Curso subregional de formación de gerentes de formación técnicoprofesional, CINTERFOR, Montevideo, pp. 59-62.

Gibb, A. (1988). Stimulating new business development. Geneva. Switzer-land: ILO. Recuperado el 13 de octubre 2014 de http://redalyc.org/articulooa?id=71612100003

Gibbons, M. (1998). Pertinencia de la educación superior en el siglo XXI. Documento presentado en la Conferencia Mundial sobre la Educación Superior de la UNESCO. Recuperado el 08 de enero del 2014 de http://www.uv.mx/departamentalizacion/lecturas\%205/pertinenciade la educación superior en el siglo XXI pdf.

González, C.H. \& Gálvez, E.J. (2008). Modelo de emprendimiento en red: aplicación de las teorías del emprendimiento a las redes empresariales. Revista Latinoamericana de Administración. No. 40, pp. 13-31. Recuperado el 03 de octubre 2014 de: http://www.redalyc.or/articulo oa? id=71612100003.

Groot, W. y Maassen, H. (2000). Education, training and employability. Applied Economics No. 32 pp. 573-581.

Hillage, J. \& Pollard, E. (1998). Employability: Developing a framework for policy analysis. Research Brief, 85. London.

Irigoin, M. y Vargas, F. (2002). Competencia laboral: manual de conceptos, métodos y aplicaciones. Montevideo: CINTERFOR/ OIT. 
Levy-Leboyer, C. (1997). Gestión por competencias. Barcelona: Gestión 2000. Recuperado el 14 de agosto del 2013 de: http://sht.com.ar/archivo/temas/competencias.htm

Lombana, J.H. (1979).A program planning approach to teacher contultation. The school Counsellor. 26 pp 163-170.

Martínez, A. y Rentería, R. (2006). Estrategias de aprendizaje para la empleabilidad en el mercado de trabajo del profesional recién egresado. Universitas Psychologica. 6 (1) 89-103. Recuperado el 14 de marzo del 2014 de http://redalyc.uamex.mx.pdf.

McClellan, D. y Burnham, D. (1973)." Power is the great motivation”, Harvard Business School. Review, USA. Marzo- Abril.

Meza, P. (2008). Las competencias que requiere el sector empresarial mexicano de los egresados universitarios. Puebla: Instituto de Investigaciones Sociales y HumanidadesBUAP.

Mertens, L. (2000). La gestión por competencia laboral en la empresa y la formación profesional: (OEI). Cinterfort. No. 12. Madrid, España.

Molina, E. (2007). La práctica profesional, componente de formación en los futuros profesionales. Investigación educativa, 11 (19) pp. 19-34. Recuperado el 13 de marzo 2014 de http://go.galegrup.com.ezproxylocal.library.nova.edu/pse/retrieve.

Monereo, C: (2003). Internet y competencias básicas. Aula de innovación educativa. No. 126, pp. 16-20.

Neffa, J.C., Panigo, D. y Pérez, P. (2000). Actividad, empleo, desempleo, conceptos y definiciones. Asociación trabajo y sociedad/CEIL PIETTE CONICET. Bs. As.

OCDE, (2005). Definición y selección de competencias clave (DeSeCo). Resumen ejecutivo. (Versión electrónica). Recuperado el 12 de noviembre del 2013 de: http://www.pisa.oecd.org/dataoecd/47/61/35070367pdf.

OCDE (1996). Exámenes de las políticas nacionales de educación. México, educación superior. OCDE, Paris.

OIT (2004). Educación, formación y aprendizaje permanente. Recomendación No. 195 de los Recursos Humanos. Ginebra Suiza. OIT.

OIT (1993). Formación profesional. Glosario de términos escogidos. Ginebra. Cinterfor (37).

Paz, H.(2007).El aprendizaje situado como una alternativa en la formación de competencias en la ingeniería. Educación en Ingeniería. (4), pp 1-13. Recuperado el 12 de abril 2014 de de http://go.galegrup.com.ezproxylocal.library.nova.edu/pse/retrieve.

Perrenoud, P. (1999). Construir competencias desde la escuela. Santiago: Dolmen 
Rascón, O. (2010). Prospectiva de la ingeniería en México y en el Mundo. Academia de Ingeniería México. México, D.F.

Real Academia de la Lengua Española. (1999). Diccionario de lengua española Recuperado el 28 de septiembre del 2013 en: http:www.competencias/definición.

Rentería, E. (2004). Empleabilidad: una lectura psicosocial. En: seminario los trabajos de la globalización. Escuela Nacional Sindical ENS, Medellín, Colombia.

Silla, I., Gracia, F.J., Peiró, J.M. (2005). Job insecurity and health-related outcomes among different types of temporary workers. Economic and industrial democracy No. 26 pp. 89-117.

Spencer, M. \& Spencer, M. (1993). Competence at work models for superior performance. John Wiley \& Sons. Inc. Nueva York.

Schumpeter, J.A. (1984). Capitalismo, socialismo y democracia. Brasil. Zahar editores.

Thijssen, J. (2000). Employability in het brandpunt. Aanzet tot verheldering van een diffuus fenomeen. Tijdschrift HRM, 1 pp 7-34.

Tobón, S. (2010). Formación integral y competencias. Pensamiento complejo, currículo, didáctica y evaluación. Bogotá: Ecoe.

Tobón, S. (2005). El concepto de competencias en la política de la calidad de la educación superior en Colombia. Un estudio hermenéutico. (Tesis doctoral). Departamento de teoría e historia de la educación. Universidad Complutense de Madrid.

Tünnermann, C. (2002). Calidad de la educación superior. París. Francia. UNESCO.

Tünnermann, C. y López, F. (2000). La educación en el horizonte del siglo XXI. Caracas, Venezuela: IESALC-UNESCO.

Van der Heijden, B. (2002). Prerrequisites to guarantee life-long employability. Personnel Review, 31 pp. 44-61.

Vargas-Hernández, J.G. (2007). La culturocracia organizacional en México. Edición electrónica gratuita. Texto completo en www.eumed.net/libros/2007b/301.

Vargas-Hernández, J.G. (2001). Las reglas cambiantes de la competitividad global en el nuevo milenio. Las competencias en el nuevo paradigma de la globalización. Revista iberoamericana de educación. Recuperado el 25 de marzo del 2014 de http://www.rioei.org/

UNESCO (1998). La educación superior en el Siglo XXI. Visión y acción. París, Francia: Ediciones UNESCO. Recuperado el 19 de noviembre del 2013 de: http://www.education.unesco.org/educ.prog/declaration_spa.htm

York, M. (2004). Employability in higher education: What it is what it is not? York: LTSN Generic Centre/ESECT. Consultado el 13 de octubre 2014 de http://heacademy.ac.uk. 